\title{
Proteomic alterations underlie an association with teratozoospermia in obese mice sperm
}

\author{
Yuanhong Peng ${ }^{1 \dagger}$, Wenzhen Zhao ${ }^{2,3 \dagger}$, Fei $\mathrm{Qu}^{1 \dagger}$, Jia Jing ${ }^{1}$, Yanqin Hu${ }^{1}$, Yue Liu ${ }^{1 *}$ (D) and Zhide Ding ${ }^{1 *}$
}

\begin{abstract}
Background: Obesity is a worldwide crisis impairing human health. In this condition, declines in sperm quality stem from reductions in sperm concentration, motility and increase in sperm deformity. The mechanism underlying these alterations remains largely unknown. This study, determined if obesity-associated proteomic expression patterns in mice sperm parallel those in spermatozoa obtained from obese humans.

Methods: An obese mouse model was established via feeding a high-fat diet (HFD). Histological analysis identified testicular morphology and a computer assisted semen analyzer (CASA) evaluated sperm parameters. Proteome analysis was performed using a label-free quantitative LC-MS/MS system. Western blot, immunohistochemical and immunofluorescent analyses characterized protein expression levels and localization in testis, sperm and clinical samples.
\end{abstract}

Results: Bodyweight gains on the HFD induced hepatic steatosis. Declines in sperm motility accompanied sperm deformity development. Differential proteomic analysis identified reduced cytoskeletal proteins, centrosome and spindle pole associated protein 1 (CSPP1) and Centrin 1 (CETN1), in sperm from obese mice. In normal weight mice, both CSPP1 and CETN1 were localized in the spermatocytes and spermatids. Their expression was appreciable in the post-acrosomal region parallel to the microtubule tracks of the manchette structure in spermatids, which affects spermatid head shaping and morphological maintenance. Moreover, CSPP1 was localized in the head-tail coupling apparatus of the mature sperm, while CETN1 expression was delimited to the post-acrosomal region within the sperm head. Importantly, sperm CSPP1 and CETN1 abundance in both the overweight and obese males decreased in comparison with that in normal weight men.

Conclusion: These findings show that regionally distinct expression and localization of CETN1 and CSPP1 is strongly related to spermiogenesis and sperm morphology maintaining. Obesity is associated with declines in the CETN1 and CSPP1 abundance and compromise of both sperm morphology in mice and relevant clinical samples. This parallelism between altered protein expression in mice and humans suggests that these effects may contribute to poor sperm quality including increased deformity.

Keywords: Obesity, Teratozoospermia, Sperm proteome, CSPP1, CETN1

\footnotetext{
* Correspondence: liuyue@shsmu.edu.cn; zding@shsmu.edu.cn

${ }^{\dagger}$ Yuanhong Peng, Wenzhen Zhao and Fei Qu contributed equally to this work.

${ }^{1}$ Department of Histology, Embryology, Genetics and Developmental Biology,

Shanghai Key Laboratory for Reproductive Medicine, Shanghai Jiao Tong

University School of Medicine, Shanghai 200025, China

Full list of author information is available at the end of the article
}

(c) The Author(s). 2019 Open Access This article is distributed under the terms of the Creative Commons Attribution 4.0 International License (http://creativecommons.org/licenses/by/4.0/), which permits unrestricted use, distribution, and reproduction in any medium, provided you give appropriate credit to the original author(s) and the source, provide a link to the Creative Commons license, and indicate if changes were made. The Creative Commons Public Domain Dedication waiver (http://creativecommons.org/publicdomain/zero/1.0/) applies to the data made available in this article, unless otherwise stated. 


\section{Background}

Obesity is a type of metabolic disease arising from an imbalance between caloric intake and metabolic expenditure. It can arise from a lack of physical exercise coupled with excessive food intake and genetic factors [1]. The worldwide size of the obese population has dramatically increased in recent years causing this disease to become a major problem impairing human health. Notably, in a 2018 report by the World Health Organization (WHO) (also in Global Health Observatory data repository), 1.9 billion overweight adults (BMI $\geq 25$; age $\geq 18$ ) were identified in 2016. In this population, male and female percentages were 39 and $40 \%$ respectively. Moreover, in this overweight population, there are over 650 million obese people $(\mathrm{BMI} \geq 30)$ and the male and female percentages were 11 and $15 \%$ respectively. In general, obesity can increase the risk of developing hypertension, diabetes, respiratory diseases and cardiovascular diseases [2-7], as well as male infertility or subfertility. $\mathrm{x}$

In the past decades, clinical studies showed that sperm quality decreases in the overweight and obese population, which is accompanied by decreases in both sperm concentration [8] and sperm motility [9, 10], abnormalities of acrosome reaction and sperm morphology, and increased sperm DNA damage $[8,11]$. Several underlying pathophysiological mechanisms can link male obesity to poor sperm quality, including endocrine abnormalities, chronic inflammation and oxidative damage. For instance, male obesity can increase the estrogen and leptin levels, and decrease testosterone levels in the serum $[12,13]$. Meanwhile, the pro-inflammatory cytokines (TNF $\alpha$, IL-1, IL-6, etc.) in the serum, testis and seminal plasma are markedly up-regulated in obesity males [14]. The resulting chronic inflammation accompanied by oxidative stress in the male reproductive tract, directly impairs spermatogenesis in the testis and sperm maturation in the epididymis $[15,16]$.

On the other hand, in contrast to these established alterations in obese males, the negative impact of obesity on sperm quality is still poorly understood at the molecular level. The comparative proteomic approach is an informative tool to evaluate sperm functional characteristics. In our previous study, we used label-free quantitative LC-MS/MS proteomic analysis to contrast expression patterns in obesity-associated asthenozoospermic and normozoospermic individuals. We identified redox regulating chaperone ERp57 and actin binding protein ACTRT2 as two potential effectors of obesityassociated asthenozoospermia [17], but there is much inter-individual variation in the human population, due in large part to individual differences in lifestyle and genomic polymorphisms. As a result, it is difficult to elucidate some definite differences in sperm proteome related to obesity. Therefore, diet induced obesity animal models are widely employed to simulate human obesity caused by consuming a high fat diet. However, relatively few proteomic studies are available evaluating the contribution made by obesity to inducing declines in sperm quality.

We describe here the results of proteomic analysis to compare the differential effects of a high fat diet (HFD) and a control diet (CD) on sperm protein expression patterns. The results show that the declines in centrosome and spindle pole associated protein 1 (CSPP1) and Centrin 1 (CETN1) expression levels in mice fed a HFD may contribute to obesity-induced male subfertility.

\section{Methods}

\section{Animals preparation and obese model establishment}

All of the animal experiments were conducted following the International Guiding Principles for Biomedical Research Involving Animals, and the research program was approved by the Ethics Committee of Shanghai Jiao Tong University School of Medicine. The male 3-weekold C57BL/6 mice were purchased from the Shanghai Laboratory Animal Center, and housed in the Animal Center of Shanghai Jiao Tong University School of Medicine. After one-week of adaptation to a normal standardized diet, the mice were then randomly divided into two groups. One group was continuously fed for 10 weeks a high-fat diet (HFD) containing $23.3 \%$ casein, $0.3 \%$ L-cysteine, $8.5 \%$ corn starch, $11.7 \%$ maltodextrin, $20.1 \%$ sucrose, $5.8 \%$ cellulose, $2.9 \%$ soybean oil, $20.7 \%$ lard, $5.2 \%$ mineral mix, $1.2 \%$ vitamin mix, and $0.3 \%$ choline bitartrate. The other control group was fed for the same period with CD containing 19\% casein, $0.2 \% \mathrm{~L}$ cysteine, $29.9 \%$ corn starch, 3.3\% maltodextrin, 33.2\% sucrose, $4.7 \%$ cellulose, $2.4 \%$ soybean oil, $1.9 \%$ lard, $4.3 \%$ mineral mix, $0.9 \%$ vitamin mix, and $0.2 \%$ choline bitartrate. Both groups had ad libitum food and water access and were maintained on a $12 \mathrm{~h}$ light and $12 \mathrm{~h}$ darkness cycle. Body weight of every animal was recorded weekly. The mice fed with CD or HFD for 10 weeks were employed for the following experiments.

\section{Assessment of sperm parameters}

The cauda epididymides separated from mice of each group were cut in pre-warmed $\left(37^{\circ} \mathrm{C}\right)$ Tyrode's Buffer (Sigma-Aldrich, USA) and then placed into a 5\% carbon dioxide incubator. After $15 \mathrm{~min}$ incubation, sperm motility, progressive motility and concentration were analyzed by computer-assisted sperm analysis (CASA) (Hamilton Thorne, USA). For teratozoospermia analysis, a sperm pellet was initially smeared on a glass slide. After reaching dryness at room temperature, the slide was fixed and stained as described in the Diff-Quick method (BRED Life Science Technology Inc., China). Then sperm morphology was observed under a microscope (Olympus, BX53, Japan) 
and the ratio of teratozoospermia was calculated in at least 200 sperm for each slide and repeated three times.

\section{Proteomic analysis}

For proteomic analysis, sperm samples were collected from caudal epididymes by centrifugation in a $45 \%$ Percoll gradient (GE Healthcare, Waukesha, WI, USA) (800 g, $20 \mathrm{~min}, 4^{\circ} \mathrm{C}$ ) and then washed thrice with PBS. Six samples from the $C D$ group and six samples from the HFD group were prepared for liquid chromatography tandem mass spectrometry (LC-MS) and performed as described [18]. All MS/MS spectra were searched using Proteome Discoverer 2.2 software against the mouse UniProt database and two missing cleavage sites were allowed. The tolerances of peptides and fragment ions were set at $6 \mathrm{ppm}$ and $0.5 \mathrm{Da}$, respectively.

\section{Histological analysis}

Tissues fixed in Bouin's solution were embedded in paraffin, and specimens were sliced into $5 \mu \mathrm{m}$ thick sections and mounted on glass slides, followed by deparaffinization and rehydration. The sectioned testicular and epididymal tissues were then stained with hematoxylin and eosin (H\&E) and observed under a microscope (Olympus BX53).

\section{Western blot analysis}

Western blot analyses were performed as modified as described before [18]. Testicular protein was separated by using $12 \%$ denaturing polyacrylamide gels after extracting and determining the concentration, then the protein was transferred to polyvinylidene difluoride (PVDF) membranes (Millipore, Germany). The membranes were blocked by using $5 \%$ bovine serum albumin (BSA) for $1 \mathrm{~h}$, then incubated at $4{ }^{\circ} \mathrm{C}$ overnight with the primary antibodies against CSPP1 (Abcam, USA; 1: 1000), CETN1 (Abcam, 1:2000), and $\beta$-actin (Abcam, 1: 5000 ), followed by incubation with secondary antibody conjugated to HRP (Abgent, San Diego, CA, USA, 1: 10000 dilution). Then enhanced chemiluminescence (Millipore, Germany) was employed to generate the signals detected by a Luminescent Image Analyzer (Image Quant LAS 4000, GE imagination at work, USA) according to the manufacturer's protocol. Western blot was repeated at least three times to confirm the results reproducibility.

\section{Immunohistochemistry (IHC) analysis}

IHC was performed using standard protocols. Paraffin sections were dewaxed and rehydrated, followed by antigen retrieval through boiling the tissue for $15 \mathrm{~min}$ in 10 $\mathrm{mM}$ citrate buffer, $\mathrm{pH}$ 6.0. Then the Histostain LAB-SA Detection kits (Invitrogen, MD, USA) were applied according to the manufacturer's instructions. Primary antibody exposure against CSPP1 (1:100 dilution) or CETN1 (1:200 dilution) and the normal IgG (control) were performed overnight at $4{ }^{\circ} \mathrm{C}$. The sections were stained using $\mathrm{DAB}$ and nuclei were counterstained with hematoxylin. Digital images were captured under a microscope (Olympus BX53).

\section{Immunofluorescence (IF) analysis}

The sperm smears were prepared and then fixed with $4 \%$ paraformaldehyde for $20 \mathrm{~min}$ at $4{ }^{\circ} \mathrm{C}$. The unspecific binding sites were blocked with 10\% BSA/PBS for 60 min at room temperature, and the sperm samples were incubated with the primary antibodies against CSPP1 (1: 200 dilution) or CETN1 (1:400 dilution) overnight at $4{ }^{\circ} \mathrm{C}$. Then Alexa Fluor 555-labeled donkey anti-rabbit secondary antibodies (1:500 dilution, Jackson ImmunoResearch) were used. Nuclei were counterstained with DAPI (Sigma-Aldrich). The fluorescence signals were detected under a laser scanning confocal microscope (Carl Zeiss LSM-510, Germany).

\section{Semen samples preparation and immunoblot analysis}

Human semen specimens, sperm parameters and relevant clinical data were obtained from Reproductive Medicine Center, Ruijin Hospital, Shanghai Jiao Tong University School of Medicine. Use of the semen samples was approved by the Ethics Committee of this institution and all experiments were performed in accordance with relevant guidelines and regulations. The clinical data of semen samples were divided into normal group $(18 \leq \mathrm{BMI}<25)$ and obese or overweight group (BMI $\geq 25$ ) according to the human BMI, and then sperm motility and morphology were calculated. All the donors ( 22 to 38 years old), both normal and obese or overweight, gave written informed consent for the use of their leftover semen samples when all IVF treatments finished, and then their semen specimens were collected. Notably, individuals having a history of long-term medication, varicocoele, and infection as indicated by a high number of leukocytes in the semen were excluded from the study. Additionally, samples that were hyperviscous and necrozoospermia (sperm viability $<70 \%$ ) were also excluded from the study.

Fresh human semen specimens were centrifuged $(800$ g, $10 \mathrm{~min}, 4^{\circ} \mathrm{C}$ ) and the sperm precipitates were dissociated in lysis buffer (6 M urea, $2 \mathrm{M}$ thiourea, and $4 \%$ CHAPS). The separated sperm proteins were stored immediately at $-80^{\circ} \mathrm{C}$ until further use. CSPP1 in sperm was detected using immunoblot analysis according to the protocol stated above.

\section{Statistical analysis}

All data were analyzed by SPSS software (SPSS Statistic 23. Chicago, IL, USA), and the data are reported as mean \pm STD. Comparisons between two groups were 
made using Student's t-test appropriately. One-way analysis of variance (ANOVA) test was used assuming a two-tail hypothesis with $P<0.05$. Differences were considered statistically different when $P<0.05$.

\section{Results}

C57BL/6 mice fed a HFD for 4 weeks gained significantly more body weight than their age-matched littermates fed a CD. This difference in body weights between the two groups developed after four-weeks on the HFD. Subsequently, these body weight differences became more and more significant for an additional 7 weeks. As expected, mice on the HFD for 10 weeks were significantly heavier than age-matched littermates on the CD $(32.25 \pm 0.37 \mathrm{~g}$ vs. $27.30 \pm 0.29 \mathrm{~g}, n=37, P<0.01$ ) (Fig. 1a).
Histological analysis of the HFD group hepatic cells contained fat vacuoles in, which is indicative of the development of a serious hepatic steatosis and fatty liver (Fig. 1b).

\section{Alteration of sperm parameters and testicular morphology in obese mice}

CASA evaluation of the sperm parameters revealed that the HFD group sperm motility $(44.80 \pm 1.21, n=10)$ and progressive motility $(20.10 \pm 1.27, n=10)$ were significantly decreased in comparison to those of the $C D$ group (percentage of sperm motility: $63.10 \pm 2.83, n=10$, $P<0.01$. Figure $2 \mathrm{a}$; percentage of progressive motility: $25.10 \pm 1.40, n=10, P<0.05$ Fig. $2 b)$. Moreover, the HFD group teratozoospermia ratio (total sperm deformity: $71.59 \pm 16.03 \%$, sperm head deformity: $29.13 \pm 5.10 \%$,

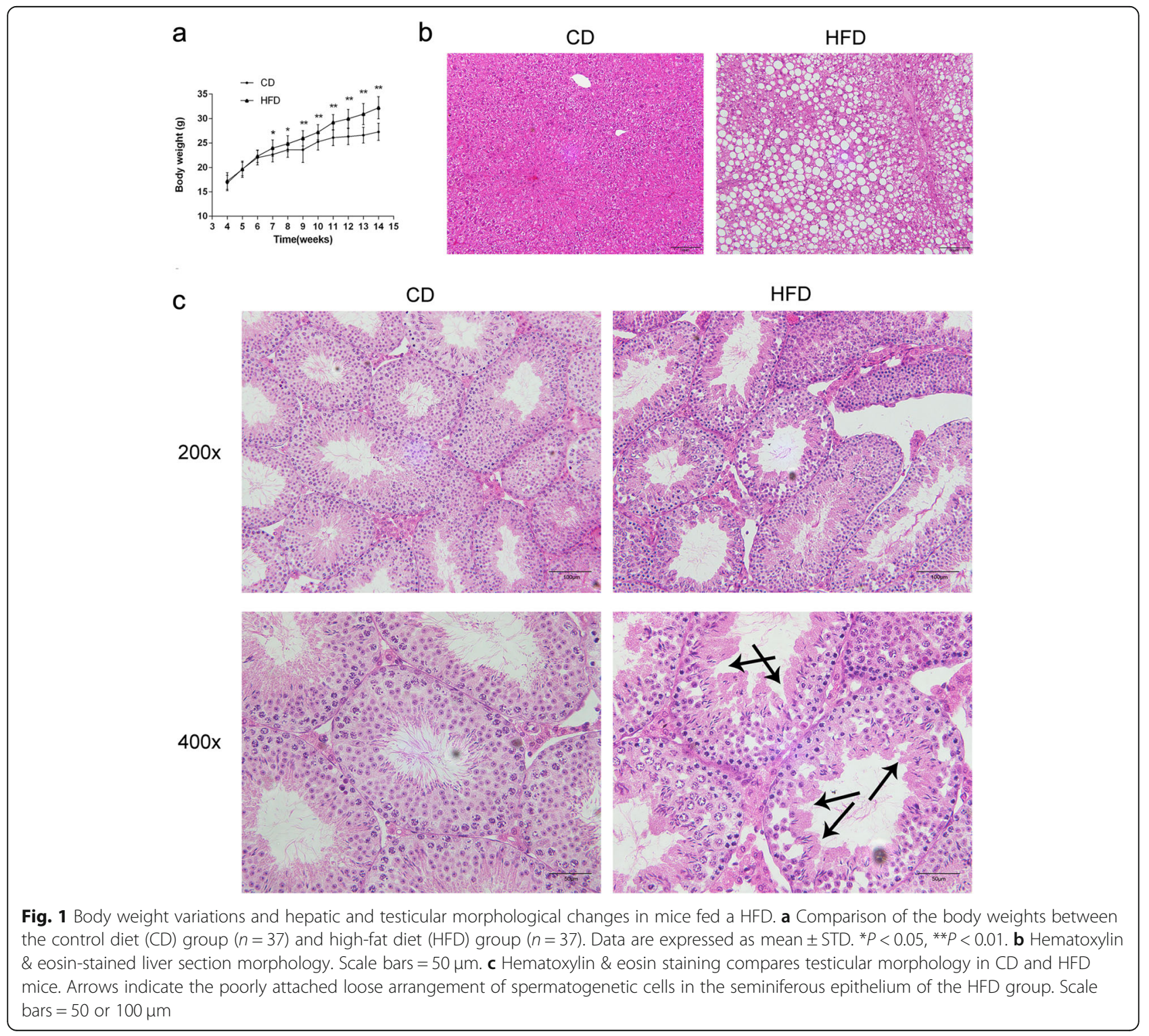




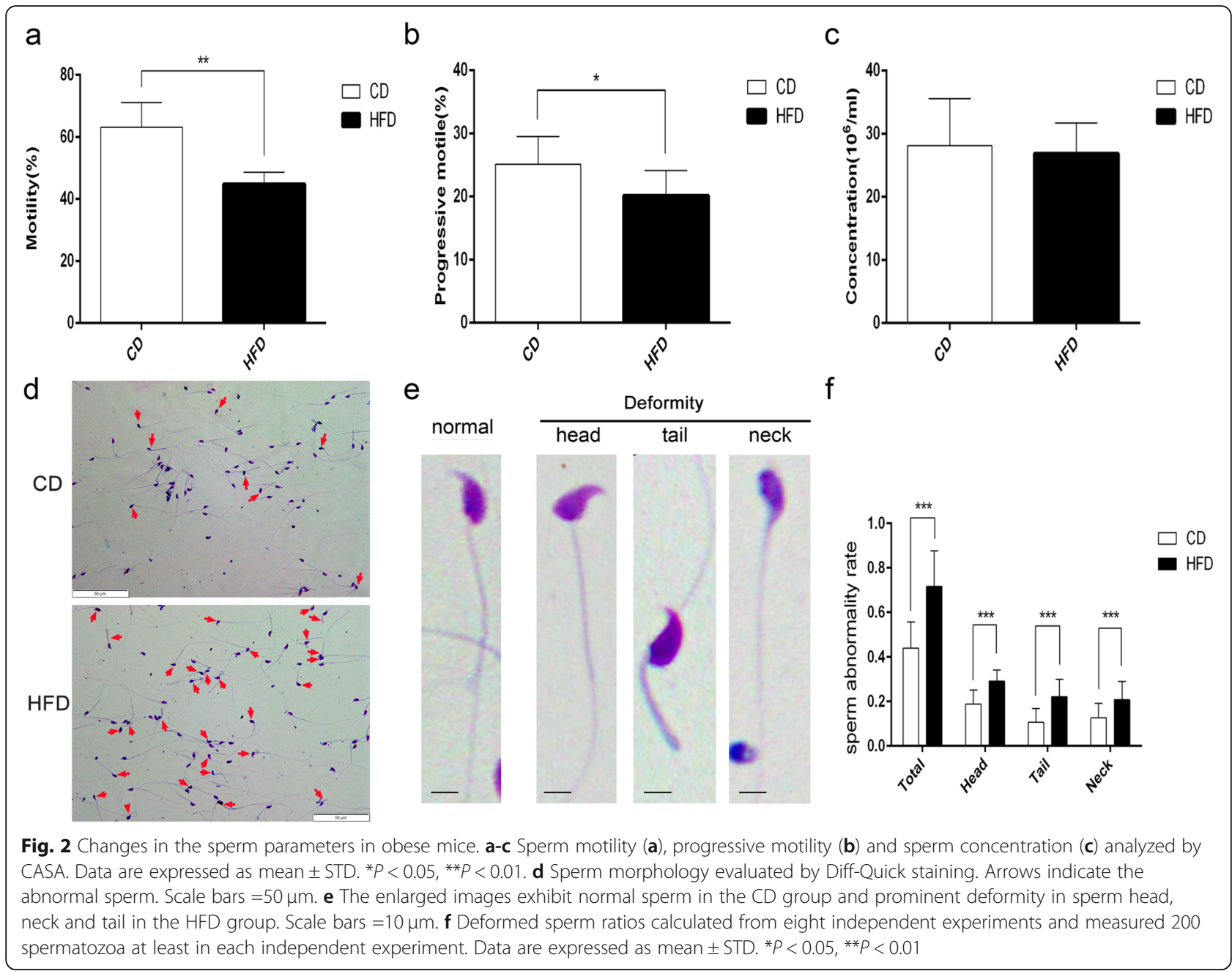

sperm tail deformity: $22.25 \pm 7.80 \%$, sperm neck deformity: $20.83 \pm 8.14 \% ; n=15$ ) was significantly larger than that of the CD group (total sperm deformity: 44.04 \pm $11.69 \%$, sperm head deformity: $18.82 \pm 6.41 \%$, sperm tail deformity: $10.67 \pm 6.25 \%$, sperm neck deformity: $12.60 \pm$ 6.54\%; $n=15, P<0.05$ Fig. $2 \mathrm{~d}-\mathrm{f})$. However, there was no difference in sperm concentration between the HFD and CD groups $(26.81 \pm 1.54$ million per $\mathrm{ml}$ vs. $28.09 \pm 2.37$ million per $\mathrm{ml}, n=10, P>0.05$ Fig. 2c).

Moreover, testicular morphological analysis showed that the HFD group morphology was disrupted compared with that in the CD group. The HFD group testicular sections had loose adhesions between the spermatogenetic cells and Sertoli cells, which disrupted spermatogenetic cell attachments and their organization (Fig. 1c).

\section{Differential sperm protein expression patterns between HFD group and CD group}

LC - MS/MS in conjunction with Maxquant analysis of 6 sperm samples selected from the CD and HFD groups provided quantitative proteomic profiles of these two groups. One thousand five hundred and sixty-two sperm proteins were identified in total (Fig. 3a), and then two samples were chosen randomly and the reproducibility of the MS analysis was determined. The results indicate that there is an excellent correction between the two groups $\left(R^{2}=0.997\right)$. The abundance of 192 proteins was significantly different between the CD and HFD groups (Fig. 3a, Additional file 1: Table S1). Principal component analysis (PCA) of the protein expression contents showed that all samples in each group had similar protein expression profiles, whereas those taken from different groups were clearly different from each other (Fig. 3b). Kyoto Encyclopedia of Genes and Genomes (KEGG) databases were employed to search for functional annotation terms (FATs) and pathways which are enriched in the proteins whose abundance is dissimilar between the two groups. The KEGG analysis revealed that most of the differentially expressed proteins were related to oxidative phosphorylation, Parkinson's disease and Alzheimer's disease, while the others were relevant to Huntington's disease, tight junction, fatty acid 


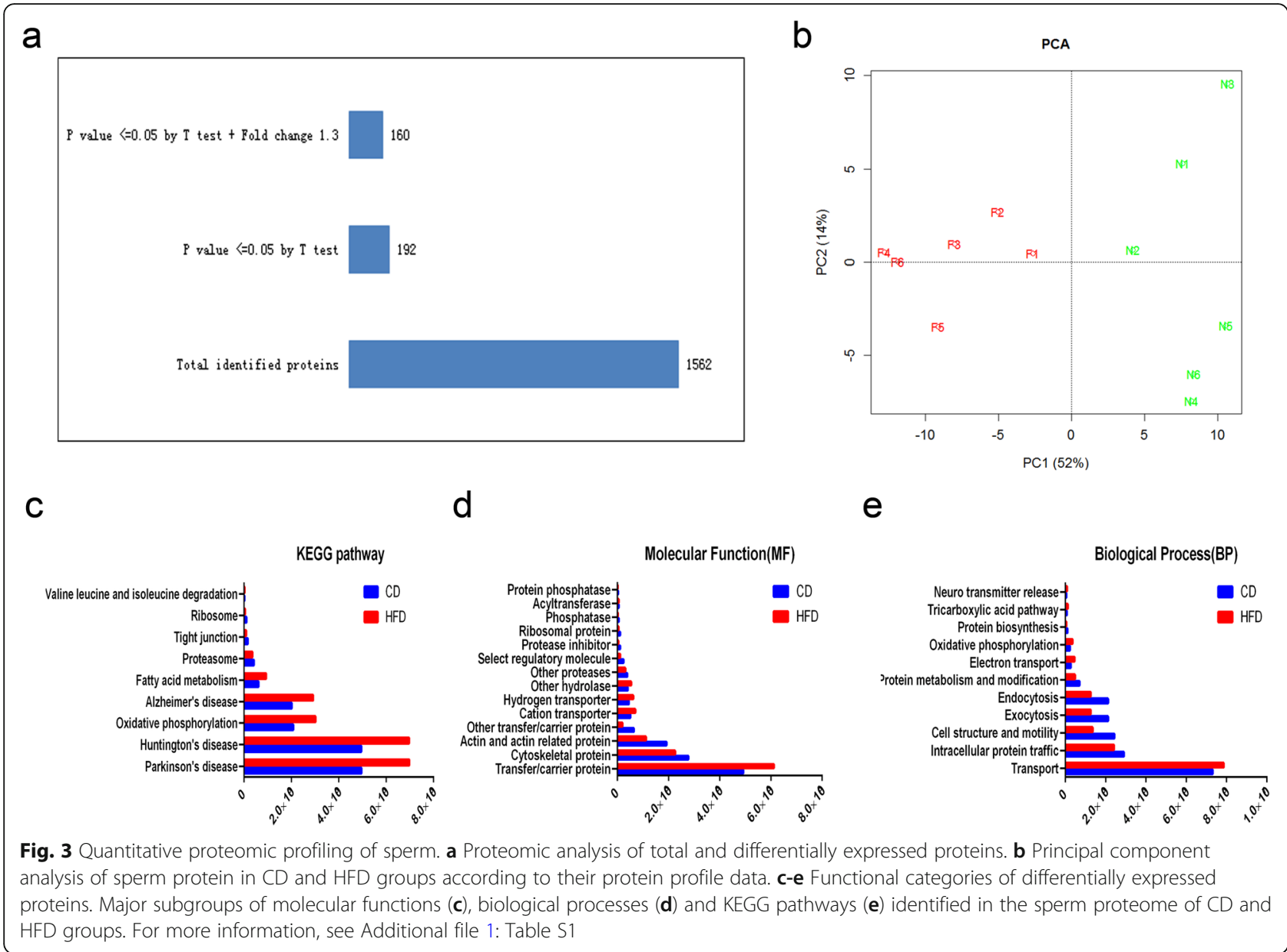

metabolism, components of proteasome mediated valine, leucine and isoleucine degradation (Fig. 3c). Gene ontology (GO) analysis of the differentially expressed proteomes showed that their functional classifications were similar to the aforementioned parameters (Fig. 3d-e).

Among these differentially expressed proteins, some are related to cytoskeleton, such as calicin [19], cylicin [19, 20], myosin [21], dynein [22, 23] and septin [24], which are thought to participate in spermiogenesis and sperm motility. More importantly, the proteomic analysis data also showed that the sperm CETN1 and CSPP1 expression levels decreased in the HFD group. CENT1 is a calmodulin-like $\mathrm{Ca}^{2+}$ binding protein which participates in spermiogenesis [25]. On the other hand, little is known about CSPP1 expression and function and its role in spermatogenesis or sperm function is unclear. These declines in CETN1 and CSPP1 expression patterns prompted us to determine if they affect sperm fertility.

\section{Expression of CSPP1 and CETN1 in mice testis and sperm} Western blot analyses showed that CSPP1 and CETN1 are widely expressed in many tissues including liver, spleen, lung, kidney, brain, testis and ovaries. The results shown in Fig. 5a indicate that their expressions are enriched in testis relative to those reported in the aforementioned tissues. Their testicular abundance suggested that they have important roles in this tissue.

IHC analysis revealed that both CSPP1 and CETN1 are visibly expressed in spermatocytes and spermatids in the seminiferous epithelium, especially around the distal half of the nucleus in the spermatids (Fig. 4a). It is known that this region consists of microtubules having an enriched manchette structure that is responsible for spermatid shaping and maintenance of sperm head morphology. IF staining specifically showed that both CSPP1 and CETN1 co-localized with $\alpha$-tubulin in the post-acrosomal region of the spermatids (Fig. 4b), suggesting their potential roles in sperm head remodeling during spermiogenesis.

Moreover, IF sperm analysis revealed that CSPP1 localized intensely in the head-tail coupling apparatus of mature sperm, and CETN1 localized in the postacrosomal region of the sperm head (Fig. 4c). All of these results suggested that these two proteins may play some important roles in spermiogenesis and maintenance of sperm morphology. 


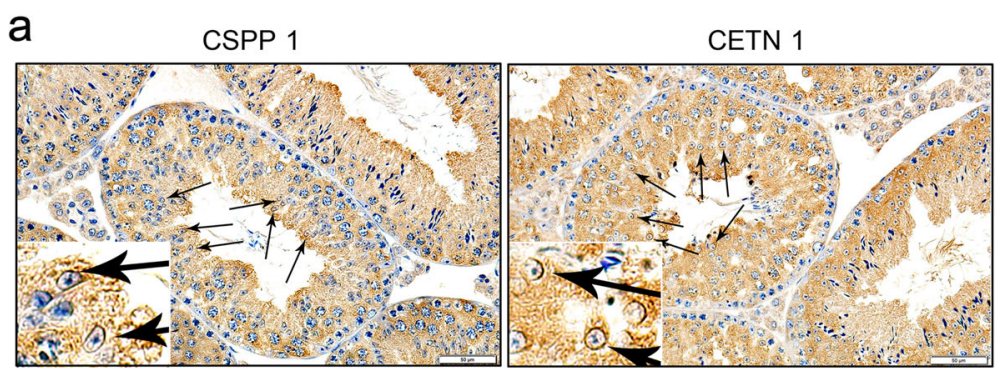

b

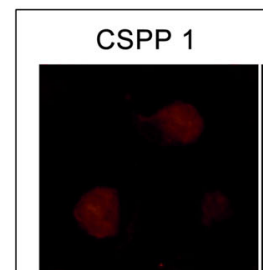

CETN 1
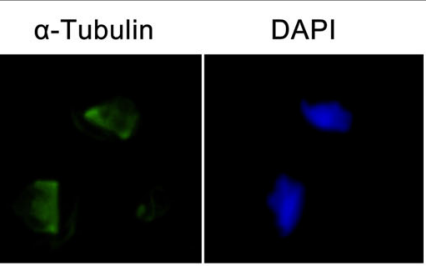

$\alpha-T u b u l i n$

DAPI
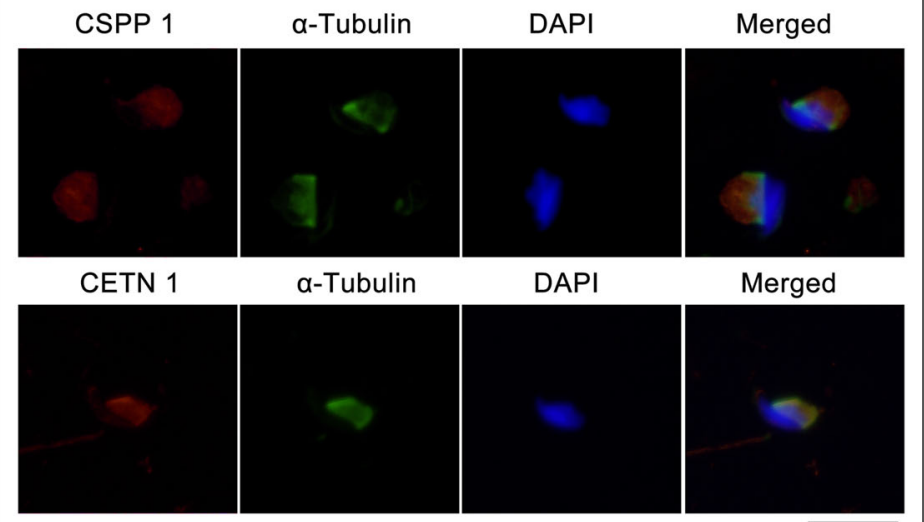

Merged

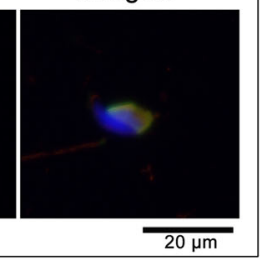

C
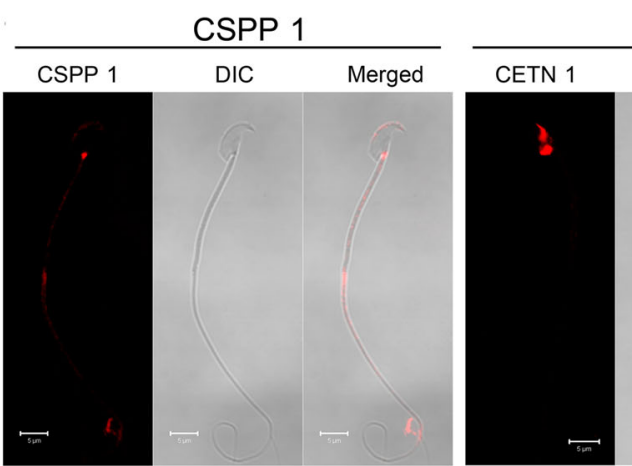

CETN 1

DIC Merged

Fig. 4 Localization of CSPP1 and CETN1 in normal mice testis and sperm. a Localization of CSPP1 and CETN1 in mice testis evaluated by immunohistochemistry staining. Arrows indicate the distribution of CSPP1 and CETN1 in spermatids. Scale bars $=50 \mu \mathrm{m}$. $\mathbf{b}$ Immunofluorescent analysis shows that CSPP1 and CETN1 co-localized with a-tubulin (indicating manchette structure) in spermatids, respectively. Scale bars $=20 \mu \mathrm{m}$. c Localization of CSPP1 and CETN1 in mice sperm. Scale bars $=5 \mu \mathrm{m}$

HFD-induced suppression of CSPP1 and CETN1 expression Western blot analyses confirmed that the HFD reduced the sperm CSPP1 and CETN1 expression levels below those in the CD group (Fig. 5b, c). Combined with their function in regulating the cytoskeletal architecture, we presumed that reduced testicular CSPP1 and CETN1 expressions levels in the HFD group contributes to their high ratio of sperm deformity.

\section{Relationship between CSPP1 expression and sperm quality in clinical samples}

Even though the function of CENT1 is known in spermatogenesis, there is scant information regarding the role of CSPP1 and CETN1 in obesity associated teratozoospermia.
To determine if CSPP1 and CETN1 expressions correlate with sperm deformity, CSPP1 expression levels were compared with sperm quality in clinical samples obtained from normal males $(\mathrm{BMI}=21.88 \pm 1.72, n=82)$ and overweight or obese males $(\mathrm{BMI}=28.43 \pm 2.82, n=190, P<0.01)$. The age of normal males $(31.78 \pm 5.79, n=82)$ and overweight or obese males $(32.73 \pm 4.91, n=190)$ was similar to each other $(P=0.273)$. The results shown in Fig. 6a compare the sperm motility and concentration of controls and overweight or obese individuals. In the overweight and obese group, sperm motility was $56.93 \pm 24.80 \%$ and sperm concentration was $84.84 \pm 61.08(n=190)$ whose values were both significantly less than those in the controls (sperm motility: $80.25 \pm 10.13$, sperm concentration: 


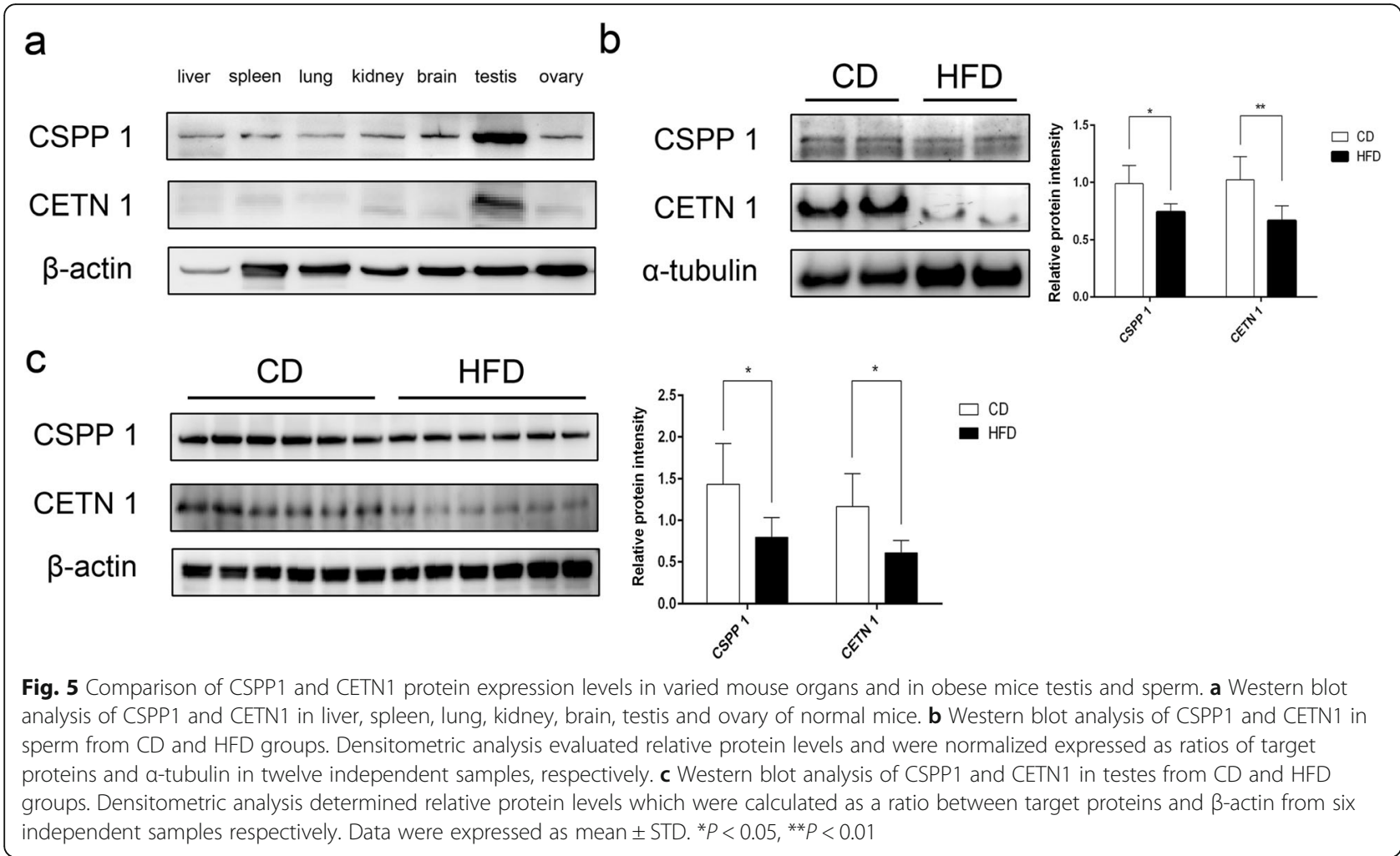

113.35 $\pm 47.19, n=82, P<0.01$ ) (Fig. 6b, c). Meanwhile, the ratio of sperm with normal morphology in overweight or obesity males $(6.77 \pm 3.66 \%, n=190)$ is significantly less than in that in normal males (9.45 $\pm 3.66 \%, n=82, P<0.01$ ) (Fig. 6d, e).

Moreover, IF analysis showed that CSPP1 and CETN1 were also localized in the head-tail coupling apparatus of human sperm (Fig. 6f), which coincides with their centrosome localization and flagellar outgrowth ability [25-27]. Additionally, immunoblot analysis showed that CSPP1 and CETN1 contents were significantly reduced in sperm from overweight or obesity males compared with that from normal males, which is in agreement with the results in the mice of HFD group (Fig. 6g). These results strongly suggest that declines in CSPP1 and CETN1 expression levels in sperm of the HFD group and clinical samples of obese males are associated with sperm deformity or teratozoospermia.

\section{Discussion}

As obesity prevalence is continuously increasing worldwide, there is accumulating evidence indicating a correlation between obesity and reduced male fertility. The results of many clinical investigations reveal that there is a possible association between obesity and the occurrence of abnormal sperm parameters. They include decreases in sperm concentration, motility and increased incidence of deformities [28, 29]. However, the underlying mechanisms are still poorly understood regarding how obesity leads to declines in sperm quality and fertility. In our previous report, the HFD mice developed a remarkable impairment of sperm function, including reduced sperm motility and abnormal sperm morphology [16]. Herein, we describe the differences in the spermatozoa proteomes between the HFD and CD groups. The results clearly show that adequate expression levels of CSPP1, a cytoskeletal related protein, is potentially related to the maintenance of normal sperm morphology.

Sperm quality is affected by pathophysiological sequelae that include altered hormone levels, chronic inflammation and generation of excessive reactive oxygen species in the reproductive system. Moreover, there is an increasing awareness that male obesity increases the risk of oligozoospermia, asthenozoospermia and teratozoospermia. Obesity is associated with an inverse relationship between increases in gene/protein expression levels and declines in sperm quality. For instance, Shi et al. [30] found that high levels of protein-tyrosine phosphatase 1B (PTP1B) expression and activity were associated with the appearance of a defect in sperm acrosome reaction (AR) in sperm of obese mice. Zhao et al. [31] reported that testicular oxidative stress in mice on a HFD was related to declines in CAT and GSH-Px activity. On the other hand, decreased Crisp4 expression in testis and epididymis in the HFD group may be a cause of declines 
a

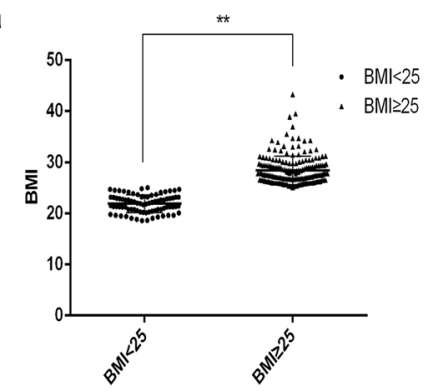

C

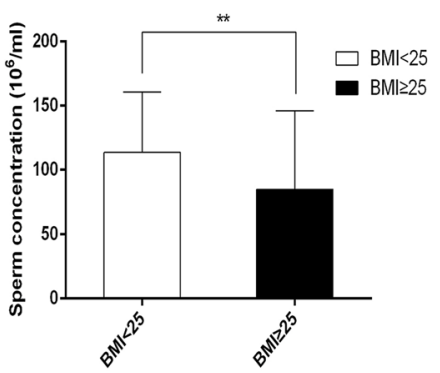

b

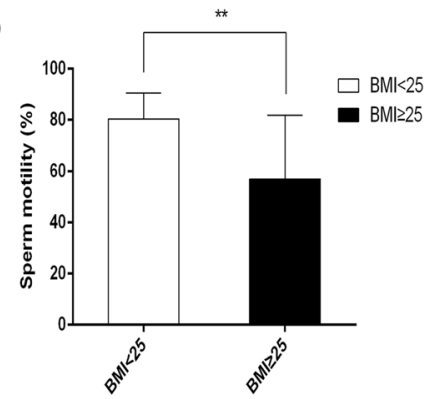

d

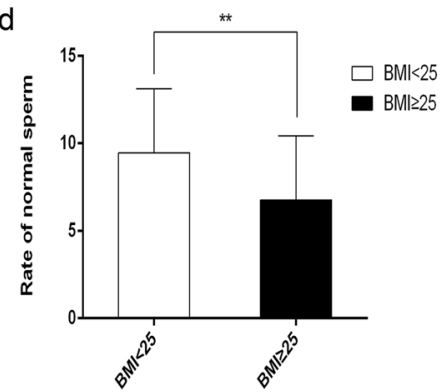

e

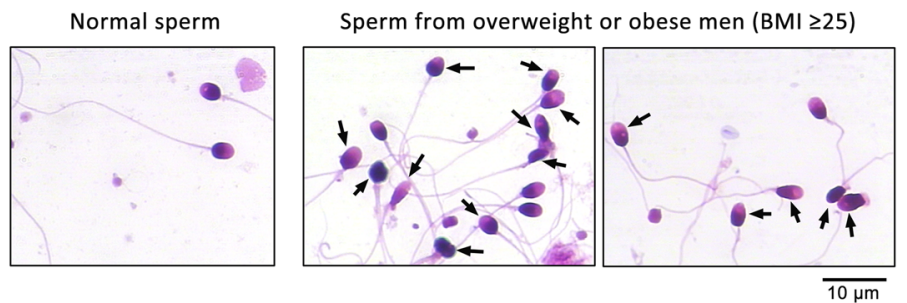

f
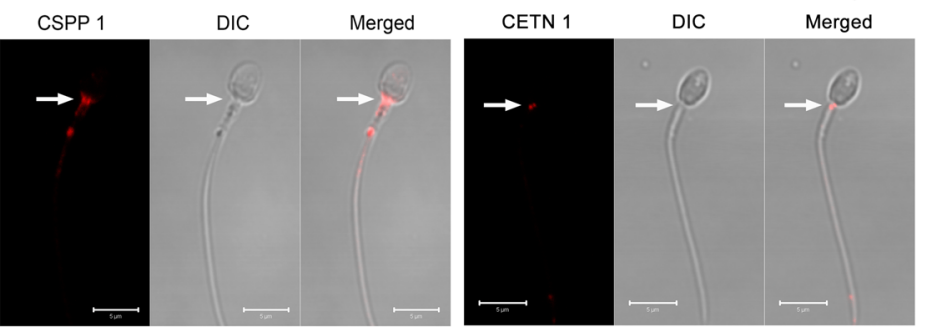

g
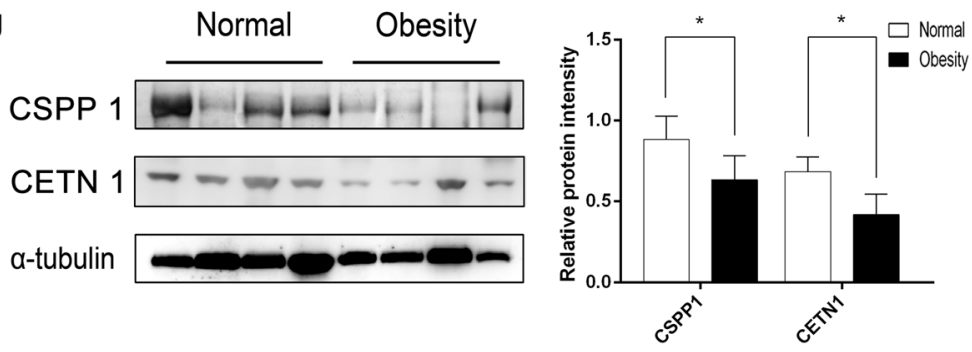

Fig. 6 Diagnostic sperm parameters of normal and overweight or obese individuals and reduced abundance of CSPP1 and CETN1 in sperm from these individuals. a-d Body mass index (BMI) (a), sperm motility (b), sperm concentration (c) and sperm deformity (d) were calculated in normal $(\mathrm{BMl}<25, n=82)$ and overweight or obese $(\mathrm{BMI} \geq 25, n=190)$ individuals respectively. Data are expressed as mean $\pm \mathrm{STD}$. ${ }^{*} P<0.01$. e Sperm morphology evaluated by Diff-Quick staining. Arrows indicate the abnormal sperm. Scale bars $=10 \mu \mathrm{m}$. $\mathbf{f}$ CSPP1 and CETN1 localization in human sperm evaluated by immunofluorescent staining. Arrows indicated the positive signals in spermatozoa. Scale bars $=5 \mu \mathrm{m}$. $\mathbf{g}$ Western blot analysis of CSPP1 and CETN1 in human sperm from normal and overweight or obese individuals, respectively. Densitometric analysis evaluated the relative protein levels by a ratio of CSPP1 or CETN1 to a-tubulin in eight independent samples, respectively. Data are expressed as mean \pm STD. ${ }^{*} P<0.05$ 
in reproductive success [32]. A previous study found that the methylation percentages at MEG3, SNRPN and SGCE/PEG10 differentially methylated regions (DMRs) significantly decreased in sperm of overweight or obese individuals. Furthermore, DNA methylation of DMRs increased on MEG3-IG and H19 in their sperm [33]. However, the obesity-induced mechanisms and key molecules are still ambiguous that contribute to poor sperm quality development.

To clarify how obesity reduces sperm quality, we addressed this question by using the described HFD model in mice $[16,34]$. There is a general consensus that HFDinduced obesity is associated with declines in sperm motility and increases in sperm deformity [14, 35-37]. Meanwhile, there is also evidence showing impaired mitochondrial activity and increased sperm DNA damage caused by increases in ROS generation in sperm of the obese mice fed a HFD [38]. On the other hand, proteomic study of sperm is a more revealing approach to clarify the identity of key factors regulating sperm quality. This procedure is the most suitable because spermatozoa released from testis are transcriptionally and translationally suppressed and their functional maturation in epididymis is completely totally dependent on post-translational modifications [39]. The proteomic approach can identify differences in sperm protein profiles between normal and obese individuals. The human sperm proteome datasets in the public domain contain 1056 proteins including Triton-X soluble and insoluble fractions [40] and 1429 proteins in the dissociated head and tail fractions [41]. Mayank et al. [42] identified 667 different proteins from normozoospermic and asthenozoospermic sperm samples, and 5 proteins which were significantly down-regulated in asthenozoospermia containing diversiform nodes related to sperm motility, such as Ninein, Fascin-3 and Plexin-B2. Mahmoud et al. [43] compared the proteins in the sperm tail from normozoospermia and asthenozoospermia, and identified 4 novel proteins, i.e. HSPA9, TUBB2B, SPANX B and ASRGL1, which were also involved in asthenozoospermia. Our previous report describing the proteomic analysis of human obese athenozoospermic sperm showed that downregulation of endoplasmic reticulum protein 57 (ERp57) and actin-binding-related protein T2 (ACTR T2) correlates with declines in sperm quality [17].

In this study, the proteomics approach analyzed sperm protein expression patterns in obese mice fed a HFD. Out of 1562 proteins that were identified, the expression levels of 192 proteins were statistically significant different between the HFD and CD groups $(P<0.05)$. Some of these downregulated proteins in the HFD group are associated with an array of functions including cell structure and motility, endocytosis, transfer/carrier protein, actin and actin related protein, and cytoskeletal architecture. Given these associations, they may be relevant to spermiogenesis, a process that transforms the morphology of the unpolarized spermatids into a uniquely shaped spermatozoon. In mammals, this remodeling change includes acrosome biogenesis, head shaping, nuclear formation, flagellum formation and the removal of residual cytoplasm. In this processes, cytoskeletal structures, such as the acroplaxome and manchette, are necessary to support spermatid remodeling and sperm function $[44,45]$. Meanwhile, endoplasmic reticulum proteins or vesicle trafficking-related proteins, such as GOPC [46], PICK1 [47], VPS54 [48], SMAP2 [49] and ATG7 [50], regulate proacrosomal vesicle transport from the Golgi to the acrosome and contribute to acrosome biogenesis and sperm head organization. Thus, the decreased expression of cytoskeletal proteins and vesicle proteins in sperm from obese mice might induce disturbed spermiogenesis, finally leading to disrupted and maladaptive sperm function. In our proteomic data, the cytoskeleton related proteins among the differentially expressed proteins in the HFD group, such as calicin [19], cylicin [19, 20], myosin [21], dynein [22, 23] and septin [24], are found to participate in spermiogenesis, maintaining sperm head shape and sperm motility. Moreover, there are still protein candidates in our proteomic data potentially valuable for further study on spermiogenesis and sperm function. For instance, secretory carrier-associated membrane proteins (SCAMP1 and SCAMP2), and VAMP-associated protein (VAPA) are involved in endomembrane dynamics and vesicle traffic [51-55], which can be conceivable candidates involved in maintaining spermiogenesis and male fertility.

Notably, we are aware of the limitation of LC-MS analysis. Such high throughput proteomics technologies only can be used as a biomarker discovery tool. Their putative identity requires validation before they can be used with confidence to resolve mechanisms underlying responses to environmental cues. Our proteomic data reveals that CSPP1 and CETN1 which are cytoskeletal proteins are the two candidates of the differentially expressed multifunctional proteins. We paid particular attention to these target proteins because one of them is CETN1, a wellcharacterized calmodulin-like $\mathrm{Ca}^{2+}$ binding protein expressed in all eukaryotic ciliated cells from yeast to mammals. It is expressed in the photoreceptor cells and other ciliated cells in rodents, including sperm [56]. It was shown that Cetn1 knock out male mice were sterile, which is associated with abnormal head morphology and reduced or absence of middle and main tail segments, indicating a crucial role for this protein in spermiogenesis [25]. Herein, this is the first report describing a relationship between CETN1 expression levels and obesityassociated asthenozoospermia and teratozoospermia. 
CSPP1 is a cytoskeletal protein related to centrosome/ microtubule cytoskeleton and spindle formation [26]. Some reports documented that a CSPP1mutation is the main cause of Joubert syndrome (JBTS), a type of invisible cilia and Jeune asphyxiating thoracic dystrophy (JATD) [27], whereas overexpression of CSPP1 in hTERT-RPE cells can result in longer cilia [57]. The loss of human CSPP1 function may affect the formation and length of primary cilia, and axonal transport of ciliary proteins, but no studies reported that it was relevant to male fertility or sperm function. Our data showed that CSPP1 is highly expressed in testis and enriched in the post-acrosomal half of the spermatids, which are situated parallel to the microtubule tracks of the manchette. To further delineate this alleged relationship between CSPP1 and obesity induced poor sperm quality, clinical semen parameters were evaluated and the results confirmed that overweight and obesity are both associated with asthenozoospermia and teratozoospermia. Furthermore, Western blot analysis verified that low CSPP1 expression accompanies obesityassociated human astheno-teratozoospermia. Additionally, CSPP1 localization in the sperm head-tail coupling apparatus also suggests that this protein may take part in sperm head shaping or flagellum formation during spermiogenesis. Therefore, reduced expression of CSPP1 in obese testis and sperm may contribute to disrupted and maladaptive cytoskeletal structure and sperm deformity. Whereas additional studies are required to understand precisely how CSPP1 expression in the spermatids involves in sperm head shaping and how obesity leads to declines in CSPP1 expression, our immediate goal was to set the stage for assessing the correlation of CSPP1with obesity associated asthenozoospermia and teratozoospermia.

\section{Conclusions}

In the HFD induced obese mice model, differential proteomic analysis identified a potential mechanism wherein changes in the CSPP1 and CETN1 cytoskeletal protein expression levels alter spermatid remodeling during spermiogenesis and underlie declines in sperm quality. Moreover, we demonstrated that CSPP1 and CETN1 is expressed in spermatocytes and spermatids in mouse testis and its distribution is related to the manchette structure that is crucial to spermatid remodeling and sperm function. Meanwhile, low CSPP1 and CETN1 expression levels are associated with human astheno-teratozoospermia in clinical samples. Taken together, these data suggest that regionally delimited expressions of CSPP1 and CETN1 are strongly associated with spermiogenesis and maintenance of normal sperm morphology whereas its deficiency in sperm may contribute to obesity-associated asthenozoospermia and teratozoospermia. These newly identified candidates may become useful functional markers for further unraveling how obesity leads to declines in sperm quality and male fertility.

\section{Supplementary information}

Supplementary information accompanies this paper at https://doi.org/10. 1186/s12958-019-0530-7.

Additional file 1: Table S1. Proteins identified in the sperm proteome.

\section{Abbreviations}

AR: Acrosome reaction; BSA: Bovine serum albumin; CASA: Computer-assisted sperm analysis; CD: Control diet; CETN1: Centrin-1; CSPP1: Cytoskeletal proteins centrosome and spindle pole associated protein 1; FATs: Functional annotation terms; GO: Gene ontology; H\&E: Hematoxylin and eosin; HFD: High-fat diet; IF: Immunofluorescence; IHC: Immunohistochemistry; JATD: Jeune asphyxiating thoracic dystrophy; JBTS: Joubert syndrome; KEGG: Kyoto encyclopedia of genes and genomes; KO: Knock-out; LCMS: Liquid chromatography tandem mass spectrometry; LC-MS/MS: Liquid chromatography-tandem mass chromatography; MMAF: Multiple morphological abnormalities of the sperm flagella; PVDF: Polyvinylidene difluoride; SPSS: Statistic package for social science; STD: Standard deviation; T test: student's t-test

\section{Acknowledgements}

The authors thank Mr. Weimin Fan (Reproductive Medicine Center, Ruijin Hospital) for his assistance in clinical data analysis. The authors are very appreciative of the support provided by Prof. Peter Reinach for his extensive and detailed support in improving the manuscript writing style.

\section{Author's contributions}

YP, WZ and FQ performed experiments, analyzed data, and prepared the manuscript. JJ performed part of molecular experiments. YH performed histological analysis. YL and ZD supervised the project and finally approved the manuscript. All authors read and approved the final manuscript.

\section{Funding}

This research project was supported by grants from the National Natural Science Foundation of China (No. 81571487, 81701503, 31560332, 81601334 and 81971437), and Science and Technology Commission of Shanghai Municipality (No.16ZR1418600).

\section{Availability of data and materials}

All data generated or analyzed during this study are included in this published article and its supplementary information files.

\section{Ethics approval and consent to participate}

All animal care and experimental protocols used in this study were approved by the institutional animal care and the ethic committee of Shanghai Jiao Tong University School of Medicine. Use of the human semen samples was approved by the Ethics Committee of Shanghai Jiao Tong University School of Medicine and all experiments were performed in accordance with relevant guidelines and regulations.

\section{Consent for publication}

Not applicable.

\section{Competing interests}

The authors declare that they have no competing interests.

\section{Author details}

'Department of Histology, Embryology, Genetics and Developmental Biology, Shanghai Key Laboratory for Reproductive Medicine, Shanghai Jiao Tong University School of Medicine, Shanghai 200025, China. ${ }^{2}$ Department of Histology and Embryology, School of Basic Medical Science, Dali University, Dali 671000, Yunnan, China. ${ }^{3}$ Institute of Reproductive Medicine, Dali University, Dali 671000, Yunnan, China.

Received: 30 April 2019 Accepted: 2 October 2019

Published online: 24 October 2019

\section{References}

1. Liu Y, Ding Z. Obesity, a serious etiologic factor for male subfertility in modern society. Reproduction. 2017;154:R123-r131. 
2. Jimenez V, Jambrina C, Casana E, Sacristan V, Munoz S, Darriba S, Rodo J, Mallol C, Garcia M, Leon X, et al. FGF21 gene therapy as treatment for obesity and insulin resistance. EMBO Mol Med. 2018;10:e8791.

3. Creatore MI, Glazier RH, Moineddin R, Fazli GS, Johns A, Gozdyra P, Matheson Fl, Kaufman-Shriqui V, Rosella LC, Manuel DG, Booth GL. Association of Neighborhood Walkability with Change in overweight, obesity, and diabetes. Jama. 2016;315:2211-20.

4. Chatterjee S, Khunti K, Davies MJ. Type 2 diabetes. Lancet. 2017;389:2239-51

5. Singh GM, Danaei G, Farzadfar F, Stevens GA, Woodward M, Wormser D, Kaptoge S, Whitlock G, Qiao Q, Lewington S, et al. The age-specific quantitative effects of metabolic risk factors on cardiovascular diseases and diabetes: a pooled analysis. PLoS One. 2013;8:e65174.

6. Afshin A, Forouzanfar MH, Reitsma MB, Sur P, Estep K, Lee A, Marczak L, Mokdad AH, Moradi-Lakeh M, Naghavi M, et al. Health effects of overweight and obesity in 195 countries over 25 years. N Engl J Med. 2017;377:13-27.

7. Bohula EA, Wiviott SD, McGuire DK, Inzucchi SE, Kuder J, Im K, Fanola CL, Qamar A, Brown C, Budaj A, et al. Cardiovascular safety of Lorcaserin in overweight or obese patients. N Engl J Med. 2018;379:1107-17.

8. Dupont C, Faure C, Sermondade N, Boubaya M, Eustache F, Clement P, Briot P, Berthaut I, Levy V, Cedrin-Durnerin I, et al. Obesity leads to higher risk of sperm DNA damage in infertile patients. Asian J Androl. 2013;15:622-5.

9. Aitken RJ, Baker MA. Causes and consequences of apoptosis in spermatozoa; contributions to infertility and impacts on development. Int J Dev Biol. 2013:57:265-72.

10. McPherson NO, Lane M. Male obesity and subfertility, is it really about increased adiposity? Asian J Androl. 2015;17:450-8.

11. Hammiche F, Laven JS, Twigt JM, Boellaard WP, Steegers EA, SteegersTheunissen RP. Body mass index and central adiposity are associated with sperm quality in men of subfertile couples. Hum Reprod. 2012;27:2365-72.

12. Cui H, Lopez M, Rahmouni K. The cellular and molecular bases of leptin and ghrelin resistance in obesity. Nat Rev Endocrinol. 2017;13:338-51.

13. Cui $X$, Jing $X$, Wu $X$, Yan M. Protective effect of resveratrol on spermatozoa function in male infertility induced by excess weight and obesity. Mol Med Rep. 2016;14:4659-65.

14. Sermondade N, Faure C, Fezeu L, Shayeb AG, Bonde JP, Jensen TK, Van Wely M, Cao J, Martini AC, Eskandar M, et al. BMl in relation to sperm count: an updated systematic review and collaborative meta-analysis. Hum Reprod Update. 2013;19:221-31.

15. Huang G, Yuan M, Zhang J, Li J, Gong D, Li Y, Zhang J, Lin P, Huang L. IL-6 mediates differentiation disorder during spermatogenesis in obesityassociated inflammation by affecting the expression of Zfp637 through the SOCS3/STAT3 pathway. Sci Rep. 2016;6:28012.

16. Fan Y, Liu Y, Xue K, Gu G, Fan W, Xu Y, Ding Z. Diet-induced obesity in male C57BL/6 mice decreases fertility as a consequence of disrupted blood-testis barrier. PLoS One. 2015;10:e0120775.

17. Liu Y, Guo Y, Song N, Fan Y, Li K, Teng X, Guo Q, Ding Z. Proteomic pattern changes associated with obesity-induced asthenozoospermia. Andrology. 2015;3:247-59.

18. Liu Y, Hu Y, Wang L, Xu C. Expression of transcriptional factor EB (TFEB) in differentiating spermatogonia potentially promotes cell migration in mouse seminiferous epithelium. Reprod Biol Endocrinol. 2018;16:105.

19. Hess $\mathrm{H}$, Heid $\mathrm{H}$, Franke WW. Molecular characterization of mammalian cylicin, a basic protein of the sperm head cytoskeleton. J Cell Biol. 1993;122:1043-52.

20. Rousseaux-Prevost R, Lecuyer C, Drobecq H, Sergheraert C, Dacheux JL, Rousseaux J. Characterization of boar sperm cytoskeletal cylicin II as an actin-binding protein. Biochem Biophys Res Commun. 2003;303:182-9.

21. Yang F, Wei Q, Adelstein RS, Wang PJ. Non-muscle myosin IIB is essential for cytokinesis during male meiotic cell divisions. Dev Biol. 2012;369:356-61.

22. Rashid S, Grzmil P, Drenckhahn JD, Meinhardt A, Adham I, Engel W, Neesen J. Disruption of the murine dynein light chain gene Tcte3-3 results in asthenozoospermia. Reproduction. 2010;139:99-111.

23. Ben Khelifa M, Coutton C, Zouari R, Karaouzene T, Rendu J, Bidart M, Yassine S, Pierre V, Delaroche J, Hennebicq S, et al. Mutations in DNAH1, which encodes an inner arm heavy chain dynein, lead to male infertility from multiple morphological abnormalities of the sperm flagella. Am J Hum Genet. 2014;94:95-104.

24. Kuo YC, Lin YH, Chen HI, Wang YY, Chiou YW, Lin HH, Pan HA, Wu CM, Su SM, Hsu CC, Kuo PL. SEPT12 mutations cause male infertility with defective sperm annulus. Hum Mutat. 2012;33:710-9.
25. Avasthi P, Scheel JF, Ying G, Frederick JM, Baehr W, Wolfrum U. Germline deletion of Cetn1 causes infertility in male mice. J Cell Sci. 2013;126:3204-13

26. Patzke S, Hauge H, Sioud M, Finne EF, Sivertsen EA, Delabie J, Stokke T, Aasheim HC. Identification of a novel centrosome/microtubule-associated coiled-coil protein involved in cell-cycle progression and spindle organization. Oncogene. 2005;24:1159-73.

27. Tuz K, Bachmann-Gagescu R, O'Day DR, Hua K, Isabella CR, Phelps IG, Stolarski AE, O'Roak BJ, Dempsey JC, Lourenco C, et al. Mutations in CSPP1 cause primary cilia abnormalities and Joubert syndrome with or without Jeune asphyxiating thoracic dystrophy. Am J Hum Genet. 2014;94:62-72.

28. Shukla KK, Chambial S, Dwivedi S, Misra S, Sharma P. Recent scenario of obesity and male fertility. Andrology. 2014;2:809-18.

29. Guo D, Wu W, Tang Q, Qiao S, Chen Y, Chen M, Teng M, Lu C, Ding H, Xia $Y$, et al. The impact of BMl on sperm parameters and the metabolite changes of seminal plasma concomitantly. Oncotarget. 2017:8:48619-34.

30. Shi L, Zhang Q, Xu B, Jiang X, Dai Y, Zhang CY, Zen K. Sustained high protein-tyrosine phosphatase $1 \mathrm{~B}$ activity in the sperm of obese males impairs the sperm acrosome reaction. J Biol Chem. 2014;289:8432-41.

31. Zhao J, Zhai L, Liu Z, Wu S, Xu L. Leptin level and oxidative stress contribute to obesity-induced low testosterone in murine testicular tissue. Oxidative Med Cell Longev. 2014;2014:190945.

32. Borges BC, Garcia-Galiano D, da Silveira C-MS, Han X, Gavrilina GB, Saunders TL, Auchus RJ, Hammoud SS, Smith GD, Elias CF. Obesity-induced infertility in male mice is associated with disruption of Crisp4 expression and sperm fertilization capacity. Endocrinology. 2017;158:2930-43.

33. Soubry A, Guo L, Huang Z, Hoyo C, Romanus S, Price T, Murphy SK. Obesityrelated DNA methylation at imprinted genes in human sperm: results from the TIEGER study. Clin Epigenetics. 2016;8:51.

34. Fan W, Xu Y, Liu Y, Zhang Z, Lu L, Ding Z. Obesity or overweight, a chronic inflammatory status in male reproductive system, Leads to Mice and Human Subfertility. Front Physiol. 2017;8:1117.

35. Fernandes GS, Arena AC, Campos KE, Volpato GT, Anselmo-Franci JA, Damasceno DC, Kempinas WG. Glutamate-induced obesity leads to decreased sperm reserves and acceleration of transit time in the epididymis of adult male rats. Reprod Biol Endocrinol. 2012;10:105.

36. Bakos HW, Mitchell M, Setchell BP, Lane M. The effect of paternal dietinduced obesity on sperm function and fertilization in a mouse model. Int J Androl. 2011;34:402-10.

37. Ghanayem BI, Bai R, Kissling GE, Travlos G, Hoffler U. Diet-induced obesity in male mice is associated with reduced fertility and potentiation of acrylamide-induced reproductive toxicity. Biol Reprod. 2010;82:96-104.

38. Rato L, Alves MG, Cavaco JE, Oliveira PF. High-energy diets: a threat for male fertility? Obes Rev. 2014;15:996-1007.

39. Baker MA, Aitken RJ. Proteomic insights into spermatozoa: critiques, comments and concerns. Expert Rev Proteomics. 2009;6:691-705.

40. Baker MA, Reeves G, Hetherington L, Muller J, Baur I, Aitken RJ. Identification of gene products present in triton $\mathrm{X}-100$ soluble and insoluble fractions of human spermatozoa lysates using LC-MS/MS analysis. Proteomics Clin Appl. 2007;: :524-32.

41. Baker MA, Naumovski N, Hetherington L, Weinberg A, Velkov T, Aitken RJ. Head and flagella subcompartmental proteomic analysis of human spermatozoa. Proteomics. 2013;13:61-74.

42. Saraswat M, Joenvaara S, Jain T, Tomar AK, Sinha A, Singh S, Yadav S, Renkonen R. Human spermatozoa quantitative proteomic signature classifies Normo- and Asthenozoospermia. Mol Cell Proteomics. 2017;16:57-72.

43. Hashemitabar M, Sabbagh S, Orazizadeh M, Ghadiri A, Bahmanzadeh M. A proteomic analysis on human sperm tail: comparison between normozoospermia and asthenozoospermia. J Assist Reprod Genet. 2015;32:853-63.

44. Wei YL, Yang WX. The acroframosome-acroplaxome-manchette axis may function in sperm head shaping and male fertility. Gene. 2018;660:28-40.

45. Toshimori $\mathrm{K}$, Ito $\mathrm{C}$. Formation and organization of the mammalian sperm head. Arch Histol Cytol. 2003;66:383-96.

46. Yao R, Ito C, Natsume Y, Sugitani Y, Yamanaka H, Kuretake S, Yanagida K, Sato A, Toshimori K, Noda T. Lack of acrosome formation in mice lacking a Golgi protein, GOPC. Proc Natl Acad Sci U S A. 2002:99:11211-6.

47. Xiao N, Kam C, Shen C, Jin W, Wang J, Lee KM, Jiang L, Xia J. PICK1 deficiency causes male infertility in mice by disrupting acrosome formation. J Clin Invest. 2009;119:802-12. 
48. Jockusch H, Holland A, Staunton L, Schmitt-John T, Heimann P, Dowling P, Ohlendieck K. Pathoproteomics of testicular tissue deficient in the GARP component VPS54: the wobbler mouse model of globozoospermia. Proteomics. 2014;14:839-52.

49. Funaki T, Kon S, Tanabe K, Natsume W, Sato S, Shimizu T, Yoshida N, Wong WF, Ogura A, Ogawa T, et al. The Arf GAP SMAP2 is necessary for organized vesicle budding from the trans-Golgi network and subsequent acrosome formation in spermiogenesis. Mol Biol Cell. 2013;24:2633-44.

50. Wang H, Wan H, Li X, Liu W, Chen Q, Wang Y, Yang L, Tang H, Zhang X, Duan $\mathrm{E}$, et al. Atg7 is required for acrosome biogenesis during spermatogenesis in mice. Cell Res. 2014;24:852-69.

51. Lindhout FW, Cao Y, Kevenaar JT, Bodzeta A, Stucchi R, Boumpoutsari MM, Katrukha EA, Altelaar M, MacGillavry HD, Hoogenraad CC. VAP-SCRN1 interaction regulates dynamic endoplasmic reticulum remodeling and presynaptic function. EMBO J. 2019:38:e101345.

52. Dong R, Saheki Y, Swarup S, Lucast L, Harper JW, De Camilli P. Endosome-ER contacts control actin nucleation and Retromer function through VAPdependent regulation of PI4P. Cell. 2016;166:408-23.

53. Toyooka K, Matsuoka K. Exo- and endocytotic trafficking of SCAMP2. Plant Signal Behav. 2009;4:1196-8.

54. Zheng JC, Tham CT, Keatings K, Fan S, Liou AY, Numata Y, Allan D, Numata $M$. Secretory carrier membrane protein (SCAMP) deficiency influences behavior of adult flies. Front Cell Dev Biol. 2014;2:64.

55. Zhang J, Castle D. Regulation of fusion pore closure and compound exocytosis in neuroendocrine PC12 cells by SCAMP1. Traffic. 2011;12:600-14.

56. Wolfrum U, Salisbury JL. Expression of centrin isoforms in the mammalian retina. Exp Cell Res. 1998;242:10-7.

57. Lee JE, Silhavy JL, Zaki MS, Schroth J, Bielas SL, Marsh SE, Olvera J, Brancati $\mathrm{F}$, lannicelli $\mathrm{M}$, Ikegami $\mathrm{K}$, et al. CEP41 is mutated in Joubert syndrome and is required for tubulin glutamylation at the cilium. Nat Genet. 2012;44:193-9.

\section{Publisher's Note}

Springer Nature remains neutral with regard to jurisdictional claims in published maps and institutional affiliations.

Ready to submit your research? Choose BMC and benefit from:

- fast, convenient online submission

- thorough peer review by experienced researchers in your field

- rapid publication on acceptance

- support for research data, including large and complex data types

- gold Open Access which fosters wider collaboration and increased citations

- maximum visibility for your research: over $100 \mathrm{M}$ website views per year

At $\mathrm{BMC}$, research is always in progress.

Learn more biomedcentral.com/submissions 